\title{
Programmable matter
}

\author{
How quantum wellstone ushered in our modern age.
}

\section{Wil McCarthy}

4 July 2100. The flick of a switch: a wall becomes a window becomes a door. Any chair becomes a hypercomputer, any rooftop a power or waste-treatment plant. We scarcely notice; programmable matter pervades our homes, our workplaces, our vehicles and environments. There isn't a city on Earth - or Mars, for that matter - that isn't clothed in the stuff from rooftop to sub-basement. But although we rarely stop to consider it, the bones of these cities - their streets, their sewers, the hearts of their telecom networks - were laid out in a time when the properties of matter were dictated exclusively by mother nature.

Imagine: if specific mechanical or electrical properties were desired, one first had to hire miners to extract appropriate elements from the Earth, then chemists and metallurgists to mix precise proportions under precise conditions, then artisans to craft the resulting materials into components, and assemble the components into products that could then be transported to the location of desired use. The inconvenience must have been staggering.

In the 22nd century, of course, any competent designer will simply define the shape and properties they require, including 'unnatural' traits such as superreflectance, refraction matching (invisibility), and centuple bonds far stronger than diamond, then distribute the configuration file to any interested users. But prior to the invention of wellstone - the earliest form of programmable matter - this would have been pure fantasy. With this in mind, we'll look back on the invention upon which, arguably, our entire civilization rests.

Consider silicon, whose oxide is the most abundant material on Earth. Humans had been making hammers and millstones out of it for millions of years, but, as it happens, silicon is also a semiconductor. The electrical properties of pure silicon are fixed by the laws of physics, but through doping - the carefully controlled introduction of impurities - its crystals can be tuned so that, for example, room-temperature electrons have a good chance of jumping up into the conduction band.

Although invaluable in the development of twentieth-century digital electronics, silicon's killer application eventually proved to be as a storage medium for electrons. The layering of doped silica in particular ways can trap conduction electrons in a membrane so thin that, from one face to the other, their behaviour as tiny quantum wave packets takes precedence over their behaviour as particles. This is called a 'quantum well'. From there, confining the electrons along a second dimension produces a 'quantum wire', and finally, with three dimensions, a 'quantum dot'.

The unique trait of a quantum dot, as opposed to any other electronic component, is that the electrons trapped in it will arrange themselves as though they were part of an atom, even though there's no atomic nucleus for them to surround. Which atom they emulate depends on the number of electrons and the exact geometry of the wells that confine them, and in fact where a normal atom is spherical, such 'designer atoms' can be turned into cubes, tetrahedra or any other shape, and filled with vastly more electrons than any real nucleus could support, to produce 'atoms' with properties that simply don't occur in nature.

Significantly, the quantum dots needn't be part of the physical structure of the semiconductor; they can be maintained just above it through a careful balancing of electrical charges. In fact, this is the preferred method, since it permits the dots' characteristics to be adjusted without any physical modification of the substrate.

Who invented wellstone remains a matter of debate: similar work was being performed in parallel, in laboratories all over the world. Regardless of whose actual idea it was, the concept itself is deceptively simple: a diffuse lattice of crystalline silicon, superfine

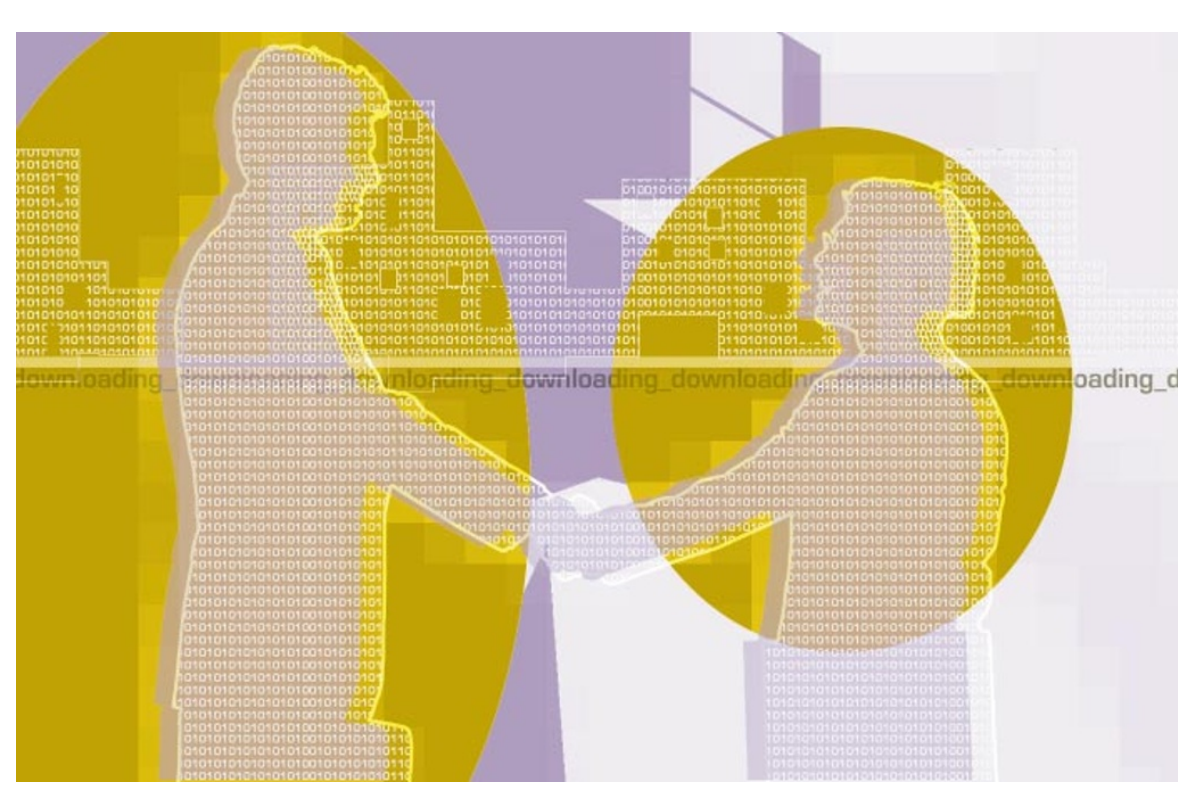

threads much thinner than a human hair, criss-crossing to form a translucent structure with roughly the density of balsa wood. Like balsa wood, the structure consists mostly of empty space, except that with the application of electrical currents, that space can be filled with 'atoms' of any desired species, producing a virtual substance with the mass of diffuse silicon, but the chemical, physical and electrical properties of some new, hybrid material.

Wellstone iron is weaker than its natural counterpart, less conductive and ferromagnetic - essentially, less 'iron-like' - and if you bash it over and over with a golf club it will gradually lose any resemblance to iron, reverting to shattered silicon and empty space. On the other hand, it's feather-light, wholly rustproof, and changeable at the flick of a bit into zinc, rubidium, or even 'imaginary' substances like impervium, the toughest superreflector known.

Of the changes wrought by programmable matter in the past century, not all have been universally welcomed. Wellstone, in the grand Promethean tradition, places the power of creation and destruction squarely into human hands. Many have argued that, far from making us strong, this power fosters a quiet corruption of spirit. Still, the fable of the three little pigs holds true - not even the Luddites among us build their houses of straw or sticks, when impervium is a free download.

Wil McCarthy, an aerospace engineer and science columnist, is the author of six science fiction novels, including Bloom (Millennium) and The Collapsium (Del Rey/Gollancz). 